\title{
Effects of gambogic acid on the activation of caspase-3 and downregulation of SIRT1 in RPMI-8226 multiple myeloma cells via the accumulation of ROS
}

\author{
LI-JING YANG* , YAN CHEN* , JING HE, SHA YI, LU WEN, SHUAI ZHAO and GUO-HUI CUI \\ Department of Hematology, Union Hospital, Tongji Medical College, \\ Huazhong University of Science and Technology, Wuhan, P.R. China
}

Received December 27, 2011; Accepted February 24, 2012

DOI: $10.3892 / 01.2012 .634$

\begin{abstract}
Multiple myeloma (MM) is the second most commonly diagnosed hematologic malignancy. Although new drugs, including bortezomib and lenalidomide, have improved the treatment landscape for MM patients, MM remains incurable. Therefore, screening for novel anti-myeloma drugs is necessary. Gambogic acid (GA), the main active ingredient of gamboges secreted from the Garcinia hanburryi tree, has been reported to exhibit potent anticancer activity in certain solid tumors and hematological malignancies, while there are few studies that are available concerning its effects on MM cells. In the present study, we investigated the anticancer activity of GA on the MM RPMI-8226 cells and further studied the underlying mechanisms by which GA affected the cells. RPMI-8226 cells were cultured and the effect of GA on cell proliferation was analyzed using MTT assay. Hoechst 33258 staining was used to visualize nuclear fragmentation, and reactive oxygen species (ROS) levels were detected. GA was found to have a significant, dose-dependent effect on growth inhibition and apoptosis induction in RPMI-8226 cells. This activity is associated with the accumulation of ROS, which contributes to the activation of caspase- 3 and the cleavage of poly (ADP-ribose) polymerase (PARP), accompanied with apoptosis in RPMI-8226 cells treated with GA. Mammalian SIRT1, as the closest homolog of the yeast Sir2, was extensively involved in regulating cell processes, including cell senescence, aging and neuronal protection, as well as having anti-apoptotic properties. Moreover, SIRT1 overexpression has
\end{abstract}

Correspondence to: Dr Guo-hui Cui, Department of Hematology, Union Hospital, Tongji Medical College, Huazhong University of Science and Technology, Jiefang Avenue 1277\#, Wuhan 430022, P.R. China

E-mail: ghcui@medmail.com.cn

*Contributed equally

Key words: gambogic acid, reactive oxygen species, SIRT1, multiple myeloma been shown to protect cancer cells from chemotherapy and ionizing radiation. In the present study, we demonstrated that GA has the potential to downregulate the expression of SIRT1 via ROS accumulation. In conclusion, our study found that GA is able to induce apoptosis in RPMI-8226 cells via ROS accumulation followed by caspase- 3 activation, PARP cleavage and SIRT1 downregulation. These results suggest that GA may have the potential to not only induce apoptosis in MM cells, but also to decrease the relapse rate of MM.

\section{Introduction}

Multiple myeloma (MM) is the second most commonly diagnosed hematologic malignancy (1). Historically, the prognosis of patients with MM has been poor due to a lack of effective therapies. Over the past decade, although the introduction of new drugs, including bortezomib and lenalidomide, has improved the treatment landscape for MM patients, almost all patients continue to experience disease relapse (2). Therefore, further investigations to find a novel anti-myeloma drug should be conducted.

Gambogic acid (GA, $\mathrm{C}_{38} \mathrm{H}_{44} \mathrm{O}_{8}$, Fig. 1) is the main active ingredient of gamboges secreted from the Garcinia hanburryi tree, which mainly grows in Southeast Asia. GA is known to have extensive antitumor activities in certain solid tumors $(3,4)$. Over the last decade, our group has confirmed that GA exhibits cytotoxicity in various types of hematological malignancy $(5,6)$. Various mechanisms by which GA exhibits extensive anticancer activity have been reported, including the downregulation of Bcl-2 (3,4), activation of p53 (7) and caspase-3 (4) and the downregulation of the HERG potassium channel (5). However, which point is the key target of GA in the induction of the apoptosis of cancer cells remains uncertain. As the changes in these proteins were correlated with the generation of reactive oxygen species (ROS) (8-11), it is suggested that GA contributes to ROS accumulation, which causes changes in the proteins mentioned above as the downstream targets of ROS.

The molecular structure of GA includes an $\alpha, \beta$-unsatured ketone, which is present in certain drugs that induce apoptosis by generating ROS (12) and ROS accumulation contributes to the apoptosis of human hepatoma SMMC-7721 cells treated 
with GA (13). Based on these theories, we hypothesized that GA has the potential to induce the apoptosis of MM RPMI8226 cells via ROS accumulation.

ROS are generally highly reactive and short-lived molecules, including superoxide anion, hydroxyl, peroxyl, alkoxyl and $\mathrm{O}_{2}$-derived non-radical species such as hydrogen peroxide that are generated by the incomplete one-electron reduction of oxygen (14). As products or by-products of cell metabolism, ROS act either as signaling molecules or as cell toxicants depending on the site of generation, spatial distribution, pulse concentration and temporal duration (9). ROS-generating enzymes are usually controlled at the gene and protein level $(15,16)$. ROS levels are balanced by non-enzymatic antioxidants (e.g., glutathione) and antioxidant enzymes (e.g., superoxide dismutase and catalase) (17). When redox signaling and control are disrupted, or the balance between oxidants and antioxidants tips towards the oxidant side, the concentration of ROS rises, contributing to the damage of biomolecules, including DNA, proteins and lipids, by oxidative modification (18). The damage that occurs may lead to disease (19), but also contributes to the anticancer activity of chemotherapy (20).

SIRT1, a NAD ${ }^{+}$-dependent deacetylase, acts on numerous substrates to control cell senescence, proliferation and apoptosis (21). There is a close association between the cellular redox status and SIRT1 function, as the overexpression of SIRT1has been reported to block oxidant-induced apoptosis via the inhibition of p53 activity (22). Under nutrient depletion, SIRT1 may protect MM RPMI-8226 cells from apoptosis (23). Moreover, SIRT1 is relevant to tumorigenesis and chemotherapy resistance (24), and SRT1720, which targets SIRT1, has been shown to inhibit growth and induce apoptosis in MM cells resistant to conventional and bortezomib therapies (25). This observation indicates that SIRT1 may be a new target in anticancer research, particularly in $\mathrm{MM}$, which has a high relapse rate.

In the present study, we examined the effect of GA on the proliferation and apoptosis of RPMI-8226 cells and explored the correlation between ROS generation, SIRT1 expression and anticancer activity. We demonstrated that GA had the potential to inhibit the proliferation of RPMI-8226 cells and induce apoptosis. These properties were mainly dependent on ROS accumulation, the activation of the downstream targets of GA and SIRT1 downregulation.

\section{Materials and methods}

Reagents and cell culture. $\mathrm{GA}\left(\mathrm{C}_{38} \mathrm{H}_{44} \mathrm{O}_{8}\right.$, molecular weight $628.75 \mathrm{~g} / \mathrm{mol}$ ), dimethyl sulfoxide (DMSO), Hoechst 33258, 3-(4,5-dimethyl-2-thiazolyl)-2,5-diphenyl-2Htetrazolium (MTT) and scavenger N-acetylcysteine (NAC) were purchased from Sigma-Aldrich (St. Louis, MO, USA). 2',7'-dichlorofluorescein-diacetate (DCFH-DA) was purchased from Beyotime (Jiangsu, China). RPMI-1640 medium was purchased from Gibco Co. (Carlsbad, CA, USA), while the fetal bovine serum (FBS) was from Hangzhou Sijiqing Biological Engineering Materials Co., Ltd. (Hangzhou, China). The Annexin V-fluorescein isothiocyanate (FITC)/propidium iodide (PI) reagent kit was purchased from Nanjing Key-Gen Biotech Co., Ltd. (Nanjing, China). The anti-activated caspase-3, anti-PARP and anti- $\gamma$-tubulin antibodies were from Cell Signaling Technology, Inc.(Danvers, MA, USA). The anti-SIRT1

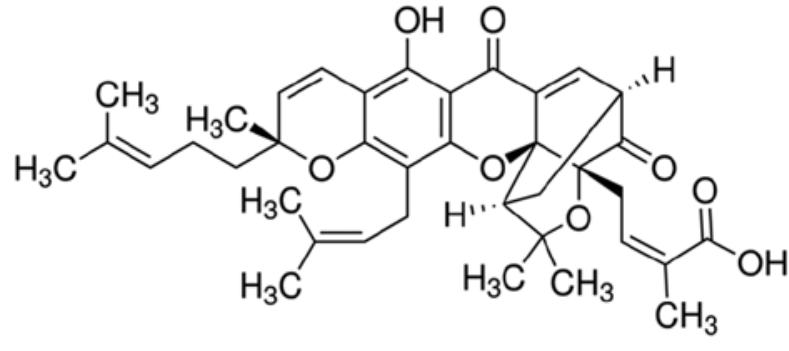

Figure 1. Molecular structure of gambogic acid.

antibody was purchased from Santa Cruz Biotechnology (Santa Cruz, CA, USA). The anti-rabbit and anti-mouse secondary antibodies were from Jackson ImmunoResearch Laboratories, Inc. (West Grove, PA, USA). The BCA Protein Assay kit, chemiluminescence reagent kit and PVDF membranes were provided by Pierce Biotechnology, Inc. (Rockford, IL, USA). Briefly, GA was dissolved in DMSO, equivalently packed, stored at $-20^{\circ} \mathrm{C}$ and thawed prior to use. The RPMI-8226 cells were donated by the Department of Immunology, Tongji Medical College, Huazhong University of Science and Technology (Wuhan, China) and were cultured in RPMI-1640 medium supplemented with 10\% FBS and placed in a humidified incubator with $95 \%$ air and $5 \% \mathrm{CO}_{2}$ at $37^{\circ} \mathrm{C}$.

MTT assay. The effect of GA on the proliferation of RPMI-8226 cells was analyzed using the MTT assay. Briefly, cells $\left(2 \times 10^{4}\right)$ were seeded in a 96-well plate and treated with $0.5,1.0,1.5,2.0$ or $2.5 \mu \mathrm{M}$ GA for $12 \mathrm{~h}$. Following incubation, $20 \mu \mathrm{l}$ MTT $(5 \mathrm{mg} / \mathrm{ml})$ was added to each well and the cells were incubated for a further $3 \mathrm{~h}$ at $37^{\circ} \mathrm{C}$. The supernatant was discarded, $150 \mu \mathrm{l}$ DMSO was added and the plate was gently agitated until the blue crystals were dissolved. Absorbance (A) at a wavelength of $490 \mathrm{~nm}$ was measured using a plate microreader (Tecan Spectra, Männedorf, Switzerland). The cell proliferation inhibition rate (\%) was calculated using the formula: [1-(A of experimental samples/A of the control)]x100.

Annexin V-FITC/PI double-labeled flow cytometry. To detect the apoptotic ratio of cells treated with GA $(1.0,1.5$ or $2.0 \mu \mathrm{M})$ alone or with NAC for $12 \mathrm{~h}$, the expression of Annexin V-FITC and the exclusion of PI were detected using two-color flow cytometry (FCM). RPMI-8226 cells were collected using EP tubes, washed twice with PBS and resuspended in $500 \mu \mathrm{l}$ binding buffer. The samples were incubated with $5 \mu 1$ Annexin V-FITC for $10 \mathrm{~min}$ at room temperature and then $5 \mu \mathrm{l}$ PI was added. Each sample was incubated for a further $10 \mathrm{~min}$ at room temperature in the dark before the fluorescence intensity was quantitated using a flow cytometer (Becton-Dickinson, Franklin Lakes, NJ, USA).

Hoechst 33258 staining. The nuclear fragmentation in RPMI-8226 cells treated with $2.0 \mu \mathrm{M}$ GA for $12 \mathrm{~h}$ was visualized using Hoechst 33258 staining. RPMI-8226 cells were plated in 12-well plates at a density of $1 \times 10^{5}$ cells/well and incubated with GA. After $12 \mathrm{~h}$, the cells were collected and washed twice in PBS. The cells were then fixed in $4 \%$ paraformaldehyde for $10 \mathrm{~min}$ at room temperature and re-suspended in PBS prior to deposition on polylysine-coated slides. After 
$30 \mathrm{~min}$, the adhered cells were permeabilized with $0.1 \%$ Triton $\mathrm{X}-100$ for $5 \mathrm{~min}$ at $4^{\circ} \mathrm{C}$ and incubated with Hoechst 33258 for $30 \mathrm{~min}$ at room temperature. Following washing with PBS, the cells were mounted with glycerol and covered with a cover slip. The images of the nuclei were captured using an Olympus BH-2 fluorescence microscope (Tokyo, Japan).

Detection of intracellular ROS levels. The levels of ROS in RPMI-8226 cells were detected using DCFH-DA. The cells were plated in 12-well plates and treated with GA at different concentrations $(1.0,1.5$ or $2.0 \mu \mathrm{M})$ in the absence or presence of $1.5 \mathrm{mM}$ NAC. When the interruption point was reached, the cells were collected and washed three times in PBS. DCFH-DA $(500 \mu \mathrm{l} ; 10 \mu \mathrm{M})$ was added to each sample and the cells were incubated at $37^{\circ} \mathrm{C}$ for $30 \mathrm{~min}$. During the incubation period, each sample was agitated every $10 \mathrm{~min}$ to ensure that the reagent reacted sufficiently with the ROS. To reduce the fluorescence background, each sample was washed twice in PBS before detecting the fluorescence intensity of DCF using FCM (Becton Dickinson).

Western blot analysis. RPMI-8226 cells treated with 1.5 or $2.0 \mu \mathrm{M}$ GA in the absence or presence of $1.5 \mathrm{mM} \mathrm{NAC}$ for $12 \mathrm{~h}$ were collected and lysed in lysis buffer $(150 \mathrm{mM} \mathrm{NaCl}, 50 \mathrm{mM}$ Tris with pH 7.4, 1\% NP40, 0.1\% SDS, 0.5 sodium deoxycholate) supplemented with protease inhibitors, followed by centrifugation at $12,000 \mathrm{x}$ g for $15 \mathrm{~min}$ at $4^{\circ} \mathrm{C}$. The protein concentration in each sample extract was detected using the BCA assay. SDS-PAGE was performed on $15 \%$ polyacrylamide gels, with $40 \mu \mathrm{g}$ of protein samples per lane. Following electrophoresis, the proteins were transferred to PVDF membranes and incubated in $5 \%$ non-fat milk at room temperature for $2 \mathrm{~h}$. Subsequently, the membranes were incubated with a specific primary antibody overnight at $4^{\circ} \mathrm{C}$. After being washed three times using PBS, the membranes were incubated with an appropriate concentration of horseradish peroxidase (HRP)-conjugated anti-mouse or anti-rabbit secondary antibody for $2 \mathrm{~h}$. After being washed a further three times with PBS, the specific protein band was visualized using the ECL kit.

Statistical analysis. Experiments were repeated three times. The data were processed using SPSS 13.0 statistical software for Windows (SPSS, Chicago, IL, USA) and shown as the mean \pm SD. Comparisons among the groups were analyzed using one-way ANOVA and the Student-Newman-Keuls (SNK) test. $\mathrm{P}<0.05$ was considered to indicate a statistically significant result.

\section{Results}

GA inhibited the proliferation of RPMI-8226 cells. The MTT assay was used to identify the effect of GA on the proliferation inhibition rate of RPMI-8226 cells. The inhibition rates of the cells treated with $0.5,1.0,1.5,2.0$ and $2.5 \mu \mathrm{M}$ were found to be $5.99 \pm 3.39,19.41 \pm 2.95,36.26 \pm 4.34,43.69 \pm 3.07$ and $52.28 \pm 1.24 \%$, respectively, which were significantly higher than those of the untreated cells (Fig. 2).

GA induced apoptosis in RPMI-8226 cells. Annexin V-FITC/ PI double-labeled FCM was applied to detect the apoptosis of

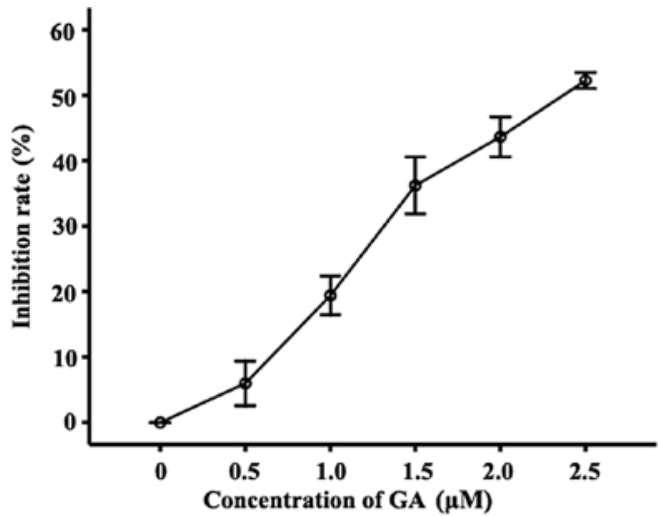

Figure 2. Effect of GA on the proliferation inhibition ratio in RPMI-8226 cells. Inhibition of the growth of RPMI-8226 cells treated with 0.5, 1.0, 1.5, 2.0 or $2.5 \mu \mathrm{M}$ GA for $12 \mathrm{~h}$ was detected by MTT assay. Compared with the untreated group, the inhibition rates of cells treated with $0.5,1.0,1.5$, 2.0 and $2.5 \mu \mathrm{M}$ GA were $5.99 \pm 3.39,19.41 \pm 2.95,36.26 \pm 4.34,43.69 \pm 3.07$ and $52.28 \pm 1.24 \%$, respectively. Data were presented as the mean \pm SD of three independent experiments. GA, gambogic acid.

RPMI-8226 cells treated with various concentrations of GA $(0,1.0,1.5$ or $2.0 \mu \mathrm{M})$ for $12 \mathrm{~h}$. The Annexin V-FITC-positive and PI-negative cells are early apoptotic cells and the Annexin V-FITC and PI-positive cells are late apoptotic cells. The total apoptotic rate is the sum of the early and late apoptotic rates. Few Annexin V-FITC-positive cells were observed in the control group (Fig. 3). However, when the concentration of GA increased to $1.0 \mu \mathrm{M}$, the Annexin V-positive cells were detected. The total apoptotic rates of RPMI-8226 cells treated with $1.0,1.5$ and $2.0 \mu \mathrm{M}$ GA were $14.10 \pm 1.51,40.23 \pm 2.63$ and $57.67 \pm 3.25 \%$, respectively, which were significantly higher than those of the control group $(4.73 \pm 0.51 \%)$.

Hoechst 33258 staining was used to detect changes in nuclear morphology in RPMI-8226 cells treated with $1.5 \mu \mathrm{M}$ GA for $12 \mathrm{~h}$. Normal RPMI-8226 cells had intact plasma membranes and ordered chromatin folding (Fig. 4). Following treatment with $1.5 \mu \mathrm{M}$ GA for $12 \mathrm{~h}$, apoptotic bodies were found to be present in the RPMI-8226 cells, in which the chromatin became condensed, the nuclear envelopes were lytic and the cytoplasm had decreased in size.

GA contributed to ROS accumulation in RPMI-8226 cells. FCM analysis of DCF fluorescence intensity was applied to monitor the level of intracellular ROS in RPMI-8226 cells treated with GA for $12 \mathrm{~h}$. The mean fluorescence intensities of DCF in RPMI-8226 cells treated with GA $(1.0,1.5$ or $2.0 \mu \mathrm{M})$ were $16.30 \pm 1.94,53.38 \pm 4.24$ and $80.42 \pm 6.48$, respectively, which were significantly higher than those of the untreated group (7.70 \pm 1.41$)$ (Fig. 5).

$N A C$ reduced the apoptosis rate in RPMI-8226 cells treated with $G A$. We applied ROS scavenger NAC to investigate the correlation between ROS accumulation and apoptosis induction in RPMI-8226 cells. As predicted, compared with the normal group, NAC did not affect the ROS level or the apoptotic rate in RPMI-8226 cells, but it significantly decreased GA-induced ROS accumulation in RPMI-8226 cells. Compared with the $2.0 \mu \mathrm{M}$ GA group, the mean DCF florescence intensity of 

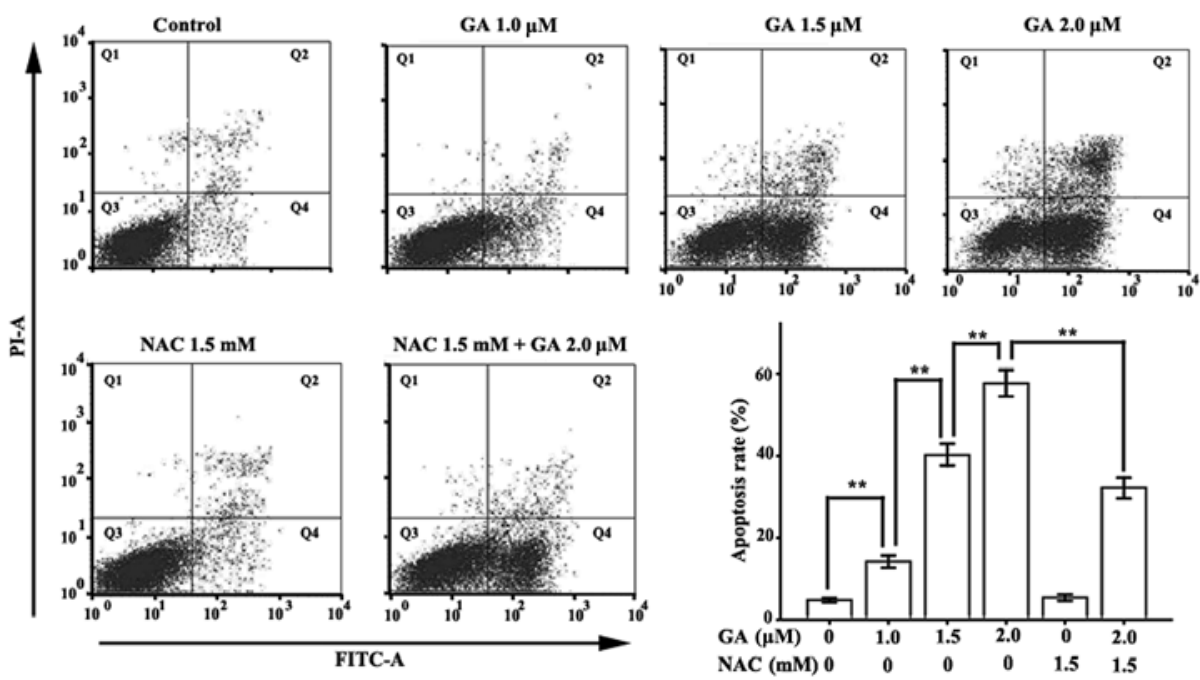

Figure 3. Assessment of apoptosis in RPMI-8226 cells treated with GA for $12 \mathrm{~h}$ in the presence or absence of NAC. Annexin V-FITC/PI double staining was used to detect the apoptotic rate. Cells in the Q2 quadrant are late apoptotic cells, while those in the Q4 quadrant are early apoptotic cells. The total apoptotic rate is the sum of the early and late apoptotic rates. Data were presented as the mean $\pm \mathrm{SD}$ of three independent experiments. ${ }^{* *} \mathrm{P}<0.05$. GA, gambogic acid; NAC, N-acetylcysteine; Annexin V-FITC/PI, Annexin V-fluorescein isothiocyanate/propidium iodide.
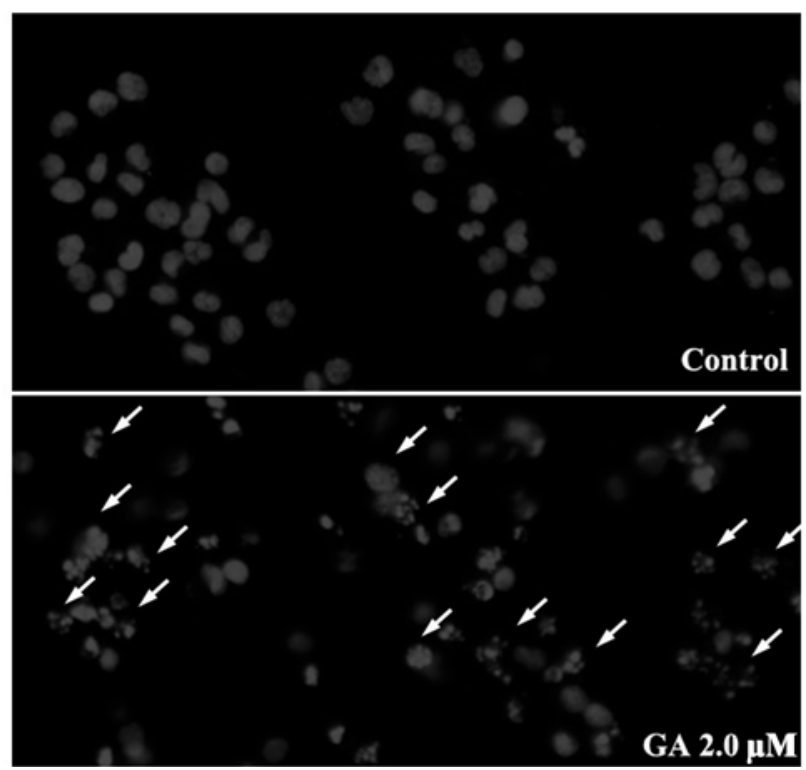

Figure 4. Effect of GA on the apoptosis of RPMI-8226 cells. RPMI-8226 cells treated with $2.0 \mu \mathrm{M}$ GA for $12 \mathrm{~h}$ were stained with Hoechst 33258 and detected using a fluorescence microscope. In the control group, the untreated cells showed normal cell nuclear morphology. Following treatment with GA for $12 \mathrm{~h}$, a number of apoptotic bodies showing fragmented or condensed nuclei (indicated by the arrows) were detected. GA, gambogic acid.

RPMI-8226 cells treated with $1.5 \mathrm{mM}$ NAC and $2.0 \mu \mathrm{M}$ GA was markedly decreased, from $80.42 \pm 6.48$ to $43.30 \pm 4.46$. The apoptotic ratio was also significantly reduced, from $57.67 \pm 3.25$ to $32.10 \pm 2.47$.

ROS accumulation leads to the activation of caspase-3 and cleavage of poly (ADP-ribose) polymerase (PARP). ROS accumulation is known to activate caspase- 3 in human hepatoma SMMC-7721 cells (13). The results of the present study indicate that GA increased the amount of activated caspase-3 in a dose-dependent manner. PARP, a $116 \mathrm{kDa}$ protein and a major substrate of activated caspase-3, is cleaved to form an amino-terminal DNA binding fragment $(24 \mathrm{kDa})$ and a carboxy-terminal catalytic fragment (89 kDa) (26). Following the activation of caspase-3, the level of the $89 \mathrm{kDa}$ fragment of PARP was increased (Fig. 6A). Consistent with the changes in the apoptotic rate and the ROS level, NAC significantly reduced the activation of caspase- 3 and the cleavage of PARP in RPMI-8226 cells treated with $2.0 \mu \mathrm{M}$ GA for $12 \mathrm{~h}$.

ROS accumulation leads to the downregulation of SIRT1. Western blot analysis was used to detect the expression of SIRT1 in RPMI-8226 cells treated with 1.5 or $2.0 \mu \mathrm{M}$ GA for $12 \mathrm{~h}$. GA was found to downregulate SIRT1 expression in a dose-dependent manner (Fig. 6B). Compared with the normal group, treatment with $1.5 \mathrm{mM}$ NAC alone did not affect the expression of SIRT1, whereas NAC significantly blocked GA-induced SIRT1 downregulation in RPMI-8226 cells. These data indicate that ROS accumulation mediates SIRT1 reduction.

\section{Discussion}

GA, the major active ingredient of gamboges secreted from the Garcinia hanburryi tree, has antitumor properties in solid tumors of various derivations in vitro and in vivo (27). The mechanisms of the anticancer activities of GA are complex and the most significant contributor has yet to be identified. At present, little is known as to how GA affects MM cells and the possible mechanisms by which it occurs, thus, further investigations should be conducted to determine these mechanisms.

Based on the theory that the structure of GA includes an $\alpha, \beta$-unsatured ketone and GA contributes to the accumulation of ROS mentioned above (13), we explored the anticancer potential of GA in MM RPMI-8226 cells and investigated its basic molecular mechanism. First, GA inhibited the proliferation rate of RPMI-8226 cells (Fig. 2). We also found that GA has the potential to induce ROS accumulation in RPMI-8226 cells in a dose-dependent manner. Following treatment with $2.0 \mu \mathrm{M}$ 

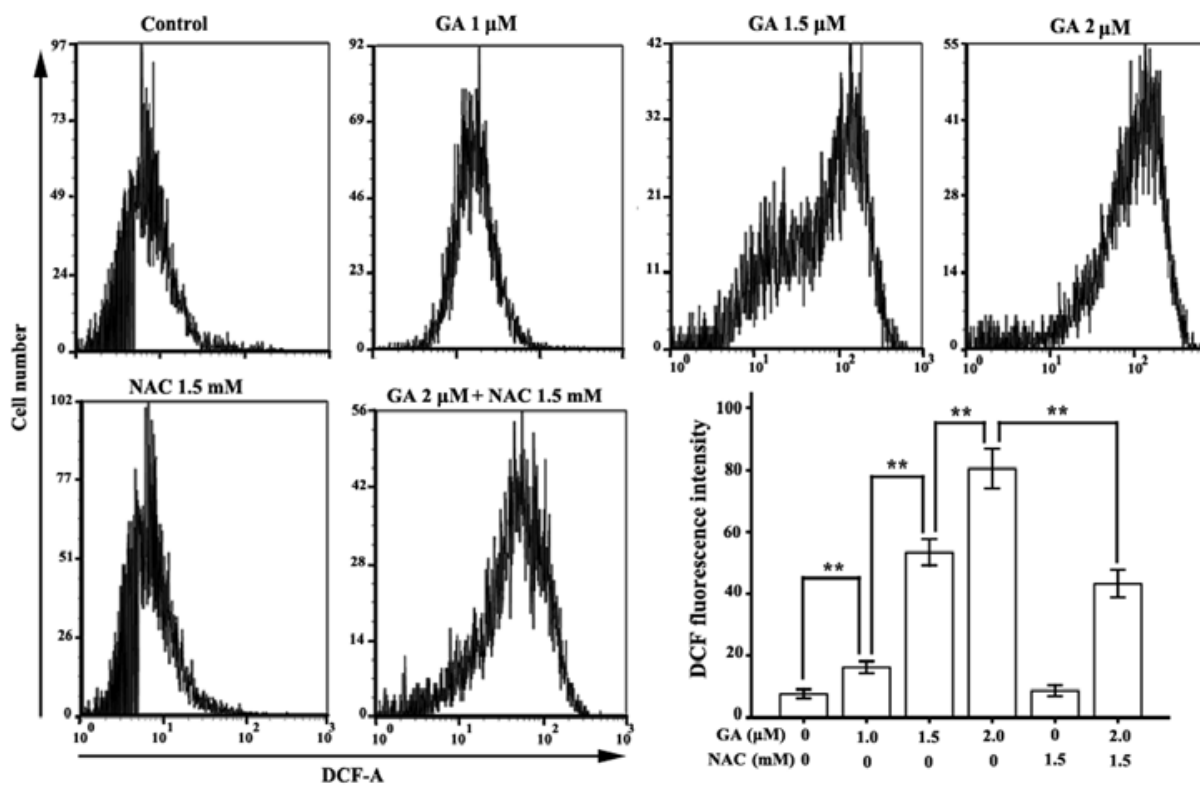

Figure 5. Assessment of the levels of intracellular ROS in RPMI-8226 cells treated with GA. Cells were incubated with various concentrations (1.0, 1.5 or $2.0 \mu \mathrm{M}$ ) of GA alone or with $1.5 \mathrm{mM} \mathrm{NAC}$ for $12 \mathrm{~h}$. DCFH was applied to detect the intracellular ROS accumulation. The mean fluorescence of DCF represents the levels of ROS. Data were presented as the mean $\pm \mathrm{SD}$ of three independent experiments. ${ }^{* *} \mathrm{P}<0.05$. GA, gambogic acid; NAC, N-acetylcysteine; ROS, reactive oxygen species; DCFH, 2',7'-dichlorofluorescein.

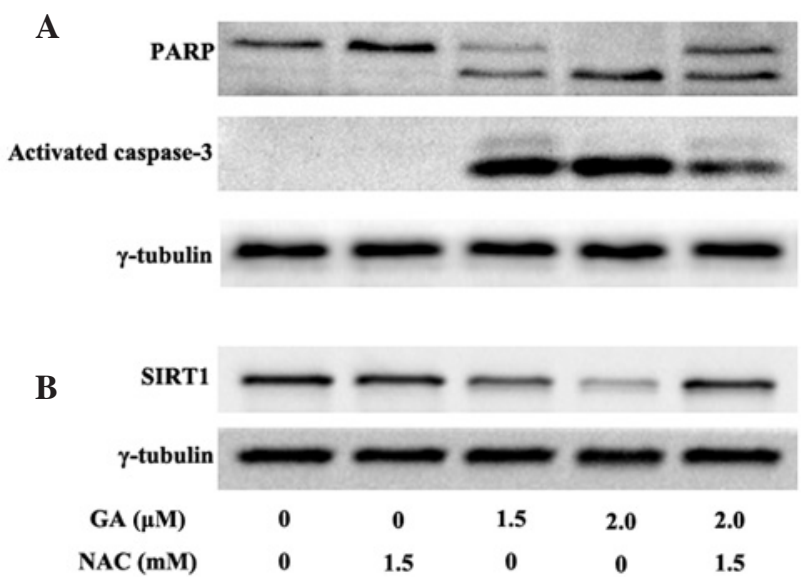

Figure 6. Effect of GA on caspase-3, PARP and SIRT1. Cells were incubated at various concentrations $(1.0,1.5$ or $2.0 \mu \mathrm{M})$ of GA alone or with $1.5 \mathrm{mM}$ $\mathrm{NAC}$ for $12 \mathrm{~h}$. Western blot analysis was used to detect the amount of activated caspase-3, PARP (the major substrate of activated caspase-3) and SIRT1. The result shows that GA contributes to the activation of caspase- 3 , followed by the cleavage of PARP in a dose-dependent manner. GA also decreases the expression of SIRT1. NAC blocks the ability of GA to activate caspase-3 and inhibit SIRT1 expression, whereas $1.5 \mathrm{mM} \mathrm{NAC}$ alone has no effect on the activation of caspase-3, cleavage of PARP, and SIRT1 downregulation in RPMI-8226 cells. GA, gambogic acid; PARP, poly (ADP-ribose) polymerase; NAC, $\mathrm{N}$-acetylcysteine.

GA for $12 \mathrm{~h}$, the mean fluorescence intensity of DCF, which represents the level of intracellular ROS, was $80.42 \pm 6.48$, which was approximately ten times that of the normal control group (7.70 \pm 1.41$)$. Consistent with this phenomenon, GA also induced the apoptosis of RPMI-8226 cells in a dose-dependent manner. The apoptosis rate of RPMI-8226 cells treated with $2.0 \mu \mathrm{M}$ GA was $57.67 \pm 3.25 \%$, which was approximately thirteen times that of the control group $(4.73 \pm 0.51 \%)$.
To confirm the correlation between GA-induced ROS accumulation and apoptosis in RPMI-8226 cells, we added ROS scavenger NAC to the GA group and detected the changes in ROS levels and apoptotic rates with or without NAC. The results showed that NAC significantly reduced the ROS accumulation induced by GA (from $80.42 \pm 6.48$ to $43.30 \pm 4.46$ ). NAC also reduced the apoptotic rate of RPMI-8226 cells treated with GA (from $57.67 \pm 3.25$ to $32.10 \pm 2.47 \%$ ). These results demonstrate that ROS accumulation is the major cause of apoptosis in RPMI-8226 cells treated with GA and that reducing ROS accumulation protects the vitality of RPMI- 8226 cells from GA treatment.

Caspase-3, as the most significant executioner caspase, induces apoptosis via the cleavage of substrates, including DNA repair- and cell cycle-related proteins, structural proteins and the mediators and regulators of apoptosis (28). ROS are known to activate caspases, which are constitutively expressed in the cytosol as inactive proenzyme monomers, via proteolysis at internal sites (29). ROS also induce the collapse of MMP, followed by the release of the pro-apoptotic factor Cyt $\mathrm{c}$ from the inner mitochondrial space to the cytosol, which in turn activates apoptosis executioner caspase-3 via the activation of apoptosis initiator caspase-9 (30). Moreover, the release of Cyt c contributes to ROS accumulation (30) and activated caspase- 3 enhances caspase- 9 processing by amplification via the promotion of caspase- 2 and -6 activation (31).

The results of the present study have shown that GA may also induce the activation of caspase- 3 in RPMI- 8226 cells in a dose-dependent manner. Since ROS scavenger NAC reduced caspase- 3 activation and the apoptotic rate, we concluded that GA induces apoptosis in RPMI-8226 cells mainly through the accumulation of ROS, which activates caspase-3. To verify this conclusion, we investigated changes in the level of PARP, which is involved in DNA repair in response to extracellular 
stress and is one of the major cleavage targets of caspase- 3 in vivo (26). Consistent with the activation of caspase-3 in RPMI-8226 cells treated with GA, the amount of $89 \mathrm{kDa}$ fragments of $116 \mathrm{kDa}$ PARP was elevated depending on the drug concentration. NAC also blocked the cleavage of PARP in cells treated with GA.

We found that GA had the potential to downregulate the expression of SIRT1 in a dose-dependent manner. Mammalian SIRT1, as the closest homolog of the yeast Sir2, is extensively involved in regulating cell processes, including cell senescence, aging and neuronal protection, as well as having anti-apoptotic properties (24). SIRT1 is upregulated in various types of cancer, including leukemia, lymphomas, soft-tissue sarcomas, prostate cancer and lung and colon carcinomas (32-35). Moreover, a high level of expression of SIRT1 has been reported to protect cancer cells from chemotherapy (36) and ionizing radiation (37). The mechanisms of SIRT1 that contribute to tumorigenesis and resistance to chemotherapy and radiotherapy are complex and include inhibitory effects on FOXO3a, p53, E2F1 and Ku70 (24). For example, SIRT1 overexpression may block oxidant-induced apoptosis via the inhibition of p53-mediated nuclear transactivation (22). The SIRT1-FOXO-3a interaction reportedly increases the transcription of stress-resistant genes and decreases the expression of FOXO-3a-dependent pro-apoptotic genes during oxidative stress (38). SIRT1 may thus be a new target in cancer therapy. To clarify, SIRT1 RNAi knockdown induced apoptosis and senescence, inhibited invasion and enhanced chemosensitivity in pancreatic cancer cells (39). SIRT1720 also inhibited growth and induced apoptosis in MM cells resistant to conventional and bortezomib therapies by targeting SIRT1 (25). In the present study, we found that GA downregulates SIRT1 expression via ROS generation, whereas NAC reduces the downregulation of SIRT1 via the elimination of the accumulation of ROS (Figs. 5 and 6B).

In conclusion, our results demonstrate that GA induces apoptosis in RPMI-8226 cells via ROS accumulation. Caspase-3, which is located downstream of ROS and executes apoptosis, was activated during the apoptosis of RPMI-8226 cells treated with GA. Moreover, high levels of ROS downregulated the expression of SIRT1, which is also relevant to apoptosis. As SIRT1 is significant in protecting cancer cells from chemotherapy, and chemotherapy resistance is the main cause of cancer relapse, we predict that GA may have the potential, not only to induce the apoptosis of MM cells, but also to decrease the relapse rate of MM.

\section{Acknowledgements}

This study was supported by the National Natural Science Foundation of China (no. 30871036/H1616 and 81070429).

\section{References}

1. Kasenda B, Ruckert A, Farthmann J, et al: Management of multiple myeloma in pregnancy: strategies for a rare challenge. Clin Lymphoma Myeloma Leuk 11: 190-197, 2011.

2. Siegel DS, Vij R and Jakubowiak AJ: Clinical roundtable monograph. Emerging treatment options for relapsed and refractory multiple myeloma. Clin Adv Hematol Oncol 9: 1-15, 2011.
3. Gu H, Rao S, Zhao J, et al: Gambogic acid reduced bcl-2 expression via $\mathrm{p} 53$ in human breast MCF-7 cancer cells. J Cancer Res Clin Oncol 135: 1777-1782, 2009.

4. Xu X, Liu Y, Wang L, et al: Gambogic acid induces apoptosis by regulating the expression of $\mathrm{Bax}$ and $\mathrm{Bcl}-2$ and enhancing caspase-3 activity in human malignant melanoma A375 cells. Int J Dermatol 48: 186-192, 2009.

5. Cui G, Shu W, Wu Q and Chen Y: Effect of Gambogic acid on the regulation of hERG channel in K562 cells in vitro. J Huazhong Univ Sci Technolog Med Sci 29: 540-545, 2009.

6. Wang Y, Chen Y, Chen Z, Wu Q, Ke WJ and Wu QL: Gambogic acid induces death inducer-obliterator 1-mediated apoptosis in Jurkat T cells. Acta Pharmacol Sin 29: 349-354, 2008.

7. Rong JJ, Hu R, Song XM, et al: Gambogic acid triggers DNA damage signaling that induces p53/p21 (Waf1/CIP1) activation through the ATR-Chk1 pathway. Cancer Lett 296: 55-64, 2010.

8. Nanduri J, Wang N, Bergson P, Yuan G, Ficker E and Prabhakar NR: Mitochondrial reactive oxygen species mediate hypoxic down-regulation of hERG channel protein. Biochem Biophys Res Commun 373: 309-314, 2008.

9. Liu B, Chen Y and St Clair DK: ROS and p53: a versatile partnership. Free Radic Biol Med 44: 1529-1535, 2008.

10. Azad N, Iyer A, Vallyathan V, et al: Role of oxidative/nitrosative stress-mediated $\mathrm{Bcl}-2$ regulation in apoptosis and malignant transformation. Ann NY Acad Sci 1203: 1-6, 2010.

11. Borutaite V and Brown GC: Caspases are reversibly inactivated by hydrogen peroxide. FEBS Lett 500: 114-118, 2001.

12. Chen YC, Shen SC and Tsai SH: Prostaglandin D(2) and J(2) induce apoptosis in human leukemia cells via activation of the caspase 3 cascade and production of reactive oxygen species. Biochim Biophys Acta 1743: 291-304, 2005.

13. Nie F, Zhang X, Qi Q, et al: Reactive oxygen species accumulation contributes to gambogic acid-induced apoptosis in human hepatoma SMMC-7721 cells. Toxicology 260: 60-67, 2009.

14. Scherz-Shouval R and Elazar Z: Regulation of autophagy by ROS: physiology and pathology. Trends Biochem Sci 36: 30-38, 2011.

15. Bokoch GM: Regulation of the human neutrophil NADPH oxidase by the Rac GTP-binding proteins. Curr Opin Cell Biol 6: 212-218, 1994.

16. Sundaresan M, Yu ZX, Ferrans VJ, et al: Regulation of reactiveoxygen-species generation in fibroblasts by Racl. Biochem J 318 (Pt 2): 379-382, 1996.

17. Terada LS: Specificity in reactive oxidant signaling: think globally, act locally. J Cell Biol 174: 615-623, 2006.

18. Jones DP: Redefining oxidative stress. Antioxid Redox Signal 8: 1865-1879, 2006.

19. Valko M, Leibfritz D, Moncol J, Cronin MT, Mazur M and Telser J: Free radicals and antioxidants in normal physiological functions and human disease. Int J Biochem Cell Biol 39: 44-84, 2007.

20. Chen Y, Jungsuwadee P, Vore M, Butterfield DA and St Clair DK: Collateral damage in cancer chemotherapy: oxidative stress in nontargeted tissues. Mol Interv 7: 147-156, 2007.

21. Smith BC, Hallows WC and Denu JM: Mechanisms and molecular probes of sirtuins. Chem Biol 15: 1002-1013, 2008.

22. Luo J, Nikolaev AY, Imai S, et al: Negative control of p53 by Sir2alpha promotes cell survival under stress. Cell 107: 137-148, 2001.

23. Zeng R, He J, Peng J, et al: The time-dependent autophagy protects against apoptosis with possible involvement of Sirt1 protein in multiple myeloma under nutrient depletion. Ann Hematol 91: 407-417, 2012.

24. Olmos Y, Brosens JJ and Lam EW: Interplay between SIRT proteins and tumour suppressor transcription factors in chemotherapeutic resistance of cancer. Drug Resist Updat 14: 35-44, 2011.

25. Chauhan D, Bandi M, Singh AV, et al: Preclinical evaluation of a novel SIRT1 modulator SRT1720 in multiple myeloma cells. Br J Haematol 155: 588-598, 2011

26. Lazebnik YA, Kaufmann SH, Desnoyers S, Poirier GG and Earnshaw WC: Cleavage of poly(ADP-ribose) polymerase by a proteinase with properties like ICE. Nature 371: 346-347, 1994.

27. Prasad S, Pandey MK, Yadav VR and Aggarwal BB: Gambogic acid inhibits STAT3 phosphorylation through activation of protein tyrosine phosphatase SHP-1: potential role in proliferation and apoptosis. Cancer Prev Res (Phila) 4: 1084-1094, 2011.

28. Degterev A, Boyce M and Yuan J: A decade of caspases. Oncogene 22: 8543-8567, 2003. 
29. Salvesen GS and Abrams JM: Caspase activation stepping on the gas or releasing the brakes? Lessons from humans and flies. Oncogene 23: 2774-2784, 2004.

30. Circu ML and Aw TY: Reactive oxygen species, cellular redox systems, and apoptosis. Free Radic Biol Med 48: 749-762, 2010.

31. Slee EA, Harte MT, Kluck RM, et al: Ordering the cytochrome c-initiated caspase cascade: hierarchical activation of caspases-2, $-3,-6,-7,-8$, and -10 in a caspase-9-dependent manner. J Cell Biol 144: 281-292, 1999.

32. Fraga MF, Agrelo R and Esteller M: Cross-talk between aging and cancer: the epigenetic language. Ann NY Acad Sci 1100: 60-74, 2007.

33. Fraga MF and Esteller M: Epigenetics and aging: the targets and the marks. Trends Genet 23: 413-418, 2007.

34. Hida Y, Kubo Y, Murao K and Arase S: Strong expression of a longevity-related protein, SIRT1, in Bowen's disease. Arch Dermatol Res 299: 103-106, 2007.
35. Lim CS: Human SIRT1: a potential biomarker for tumorigenesis? Cell Biol Int 31: 636-637, 2007.

36. Chu F, Chou PM, Zheng X, Mirkin BL and Rebbaa A: Control of multidrug resistance gene mdr1 and cancer resistance to chemotherapy by the longevity gene sirt1. Cancer Res 65: 10183-10187, 2005.

37. Matsushita N, Takami Y,Kimura M, et al: Role of NAD-dependent deacetylases SIRT1 and SIRT2 in radiation and cisplatin-induced cell death in vertebrate cells. Genes Cells 10: 321-332, 2005.

38. Brunet A, Sweeney LB, Sturgill JF, et al: Stress-dependent regulation of FOXO transcription factors by the SIRT1 deacetylase. Science 303: 2011-2015, 2004.

39. Zhao G, Cui J, Zhang JG, et al: SIRT1 RNAi knockdown induces apoptosis and senescence, inhibits invasion and enhances chemosensitivity in pancreatic cancer cells. Gene Ther 18: 920-928, 2011. 